\title{
A Bayesian inference approach: estimation of heat flux from fin for perturbed temperature data
}

\author{
HARSHA KUMAR and GNANASEKARAN NAGARAJAN*
}

Department of Mechanical Engineering, National Institute of Technology Karnataka, Mangalore 575025, India e-mail: harsha84.nitk@gmail.com; gnanasekaran@nitk.edu.in

MS received 5 April 2016; revised 2 May 2017; accepted 7 September 2017; published online 16 April 2018

\begin{abstract}
This paper reports the estimation of the unknown boundary heat flux from a fin using the Bayesian inference method. The setup consists of a rectangular mild steel fin of dimensions $250 \times 150 \times 6 \mathrm{~mm}^{3}$ and an aluminium base plate of dimensions $250 \times 150 \times 8 \mathrm{~mm}^{3}$. The fin is subjected to constant heat flux at the base and the fin setup is modelled using ANSYS14.5. The problem considered is a conjugate heat transfer from the fin, and the Navier-Stokes equation is solved to obtain the flow parameters. Grid independence study is carried out to fix the number of grids for the study considered. To reduce the computational cost, computational fluid dynamics (CFD) is replaced with artificial neural network (ANN) as the forward model. The Markov Chain Monte Carlo (MCMC) powered by Metropolis-Hastings sampling algorithm along with the Bayesian framework is used to explore the estimation space. The sensitivity analysis of the estimated temperature with respect to the unknown parameter is discussed to know the dependency of the temperature with the parameter. This paper signifies the effect of a prior model on the execution of the inverse algorithm at different noise levels. The unknown heat flux is estimated for the surrogated temperature and the estimates are reported as mean, Maximum a Posteriori (MAP) and standard deviation. The effect of a-priori information on the estimated parameter is also addressed. The standard deviation in the estimation process is referred to as the uncertainty associated with the estimated parameters.
\end{abstract}

Keywords. Mild steel fin; heat flux; ANN; Bayesian inference; MCMC; standard deviation.

\section{Introduction}

Majority of the heat transfer problems are direct. The effort is to find the temperature distribution with thermophysical properties and boundary conditions being known. These problems are classified as well-posed problems for which a solution exists; it is unique and also stable for small changes in input. In many thermal engineering applications, the temperature distribution is known, thermophysical properties and boundary heat flux causing the temperature distribution are not known; such an approach is called parameter estimation. These problems are often termed as inverse heat transfer problems. Generally, inverse problems are ill posed. Beck et al [1] provide the importance and practical nature of the inverse heat conduction problems. The inverse heat transfer problems depend on temperature and/or heat flux measurements for the estimation of unknown parameters in a heat transfer processes. Qzisik and Orlande [2] deal with fundamental concepts of inverse heat transfer problems. They have discussed extensively the concept of parameter estimation and function estimation approach.

*For correspondence
Estimation methods can be classified into two types: (1) deterministic and (2) stochastic. Because of ill-posed nature of the problem, stochastic method is commonly used. When the cause is known and the effect is observed, then the method is called as Forward model. A general parameter estimation procedure is depicted in figure $1 . Y$ represents experimental or surrogated temperature and $Z(x)$ represents temperature data from the forward model for the assumed input $x . \mathrm{R}^{2}$ is the error between the forward model temperature and experimental/surrogated temperature and generally represented as the L2 Norm. When the error is within the limit as per the error proposed, the estimation process is terminated and the solution to the problem is the initial guess, which is given as input to the forward model. In accordance to this, when the calculated error is not within the limit, then a suitable inverse model is incorporated and the objective function is minimised. Such an estimation procedure is shown in figure 2 .

Many studies have been carried out in the area of inverse heat transfer and several methods are proposed for determining the boundary heat flux. Mota et al [3] considered one-dimensional nonlinear heat conduction equation and simultaneously estimated volumetric heat capacity, thermal conductivity and boundary heat flux using the Bayesian 


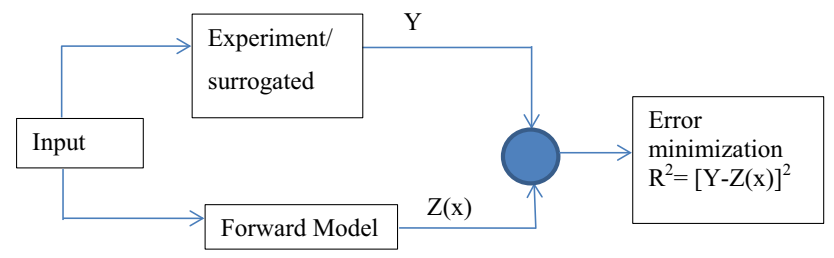

Figure 1. Flow chart of parameter estimation.

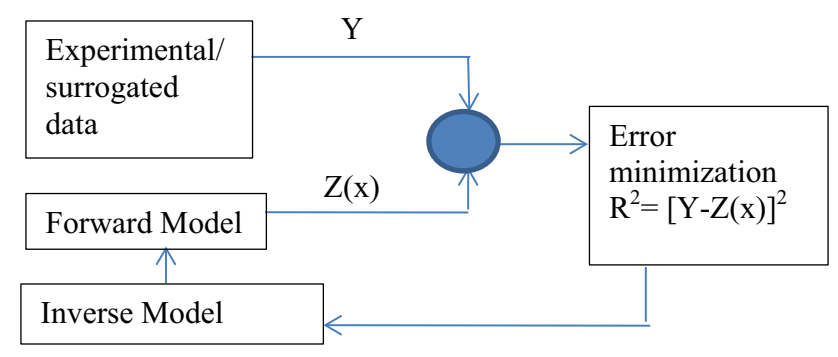

Figure 2. Parameter estimation using inverse model.

approach. Markov Chain Monte Carlo (MCMC) approach along with Metropolis-Hastings algorithm was employed. Wang and Zabaras [4] used Bayesian inference for the solution of inverse heat conduction problem. The posterior probability density function is obtained for the unknown heat flux using MCMC sampling algorithm. Giralomi [5] proved that the Bayesian influential framework provides consistent approach in the estimation of unknown parameters. Liang et al [6] considered one-dimensional transient heat conduction problem and estimated the Robin coefficient using Bayesian inference. MCMC algorithm was used to sample the unknown parameters, and it proved that the Bayesian inference method could provide accurate estimates as well as uncertainty for the problem chosen. Parthasarathy and Balaji [7] applied Bayesian inference to estimate multiple parameters in two-dimensional conduction. The focus of the work was to determine the effect of noise and prior information on the retrieval results.

Gnanasekaran and Balaji [8] used the Bayesian approach to estimate thermophysical parameters from simple, inexpensive experiments on natural convection heat transfer from a vertical rectangular fin. MCMC method was used to estimate unknown heat transfer coefficient and thermal conductivity with and without subjective priors. Konda Reddy et al [9] carried out transient experiments on a rectangular fin and obtained temperature data using the transient Liquid Crystal Thermography (LCT) technique. Treating the inverse problem as an estimation problem, the difference between the temperature measured using LCT and simulated temperatures was minimised with the Bayesian methodology in the inverse model to determine the point estimates. Cheung and Beck [10] investigated hybrid Monte Carlo method to show that it can be used to solve higher-dimensional Bayesian model updating problems. New formulae for Markov chain convergence assessment are derived. Practical issues for the feasibility of the hybrid Monte Carlo method to such problems were addressed, and improvements were proposed to make it more effective and efficient for solving such model updating problems. The effectiveness of the proposed approach for Bayesian model updating of structural dynamic models with many uncertain parameters was illustrated with a simulated data example. Gnanasekaran and Balaji [11] carried out transient experiments using fin to simultaneously estimate the unknown parameters and applied the MCMC method. The usefulness of priors was also investigated.

Balaji and Tamanna Padhi [12] used ANN in conjunction with MCMC method to solve an inverse heat conduction problem. They considered steady-state two-dimensional heat conduction from a square slab with uniform volumetric internal heat generation. They estimated heat transfer coefficient, heat generation and thermal conductivity for the surrogated data with noise and without noise. Deng and Hwang [13] used continuous-time analogue Hopfield neural network to compute the temperature distribution in forward heat conduction problems and solved inverse heat conduction problems by using a back propagation neural (BPN) network to identify the unknown boundary conditions. Ghadimi et al [14] incorporated an inverse algorithm based on the ANNs and the sequential function specification method was successfully applied for estimation of the heat flux absorbed by the locomotive brake disc. The threedimensional direct problem involving turbulent, unsteady and conjugate heat transfer boundary condition is numerically solved for known values of 47 different heat fluxes, and temperature histories of 18 different locations inside the brake disc were obtained. Somasundharam and Reddy [15] used MH-MCMC sampling algorithm in Bayesian inverse framework and estimated thermal conductivity, heat transfer coefficient and emissivity. They used Parallel Tempering (PT) and Evolutionary Monte Carlo (EMC) along with MH-MCMC to sample through correlated posterior probability density function (PPDF) to retrieve the three mentioned thermal properties. They concluded that at high noise levels, Parallel Tempering (PT) and Evolutionary Monte Carlo (EMC) perform equally and estimate the parameter with the deviation of maximum 9\%. Sensitivity analysis [15-19] is an important study that indicates the effectiveness of the change in parameter estimated on the temperature. Sensitivity analysis has control over the selection of the inverse method. Gradient-based methods are also used as the inverse model in estimating the parameters. These methods have a tendency of ending in local minima or maxima. Refs. [20,21] have estimated heat flux using different gradient-based methods and reported the values. Chen et al [22] used regression model to estimate heat flux and temperature in a multi-layer gun barrel. 
Recursive least squares estimator (RLSE) was used to extract the time-varying heat flux on-line as the input. Refs. $[23,24]$ used inverse heat conduction problem (IHCP) to estimate transient heat flux. Man [25] used finite volume method for determining the radiative heat flux calculated from the radiative heat exchange within the furnace modelled by considering the effect of furnace wall, slab, and combustion gases introduced as the boundary condition of the transient conduction equation of the slab.

Based on the literature review, the estimation of thermophysical properties, unknown heat source, heat transfer coefficients and unknown boundary conditions has become very important in the field of thermal engineering. It has been observed that Bayesian inference provides a coherent framework to characterise the unknown quantities. It is pertinent to mention here that very few literatures are available in the estimation of boundary conditions for conjugate heat transfer problems. The objective of the present paper is to estimate the boundary heat flux for a conjugate heat transfer from fin. The computational cost is reduced by implementing ANN as the forward model. MCMC, along with Metropolis-Hastings method, is used as an inverse algorithm to estimate the unknown parameter.

\section{Forward model}

The problem considered includes a steel fin placed vertically on an aluminium base. The dimension of the aluminium base is $250 \times 150 \times 8 \mathrm{~mm}^{3}$ and the base is subjected to constant heat flux. The fin dimension is $250 \times 150 \times 6 \mathrm{~mm}^{3}$. To study the convection effect, an extended domain is modelled and air is considered as the medium. The thermophysical properties are considered to be constant.

The problem is modelled as a two-dimensional conjugate heat transfer problem. In accordance with this, flow is symmetry and half of the physical domain is modelled to save the computational cost. The domain is modelled and meshed using ANSYS 14.5 tool. Meshing is done using quadrilateral cells as the element. Grid independence is applied to fix the number of grids, which is evident from table 1.

At the solid-liquid interface, inflation layers are used for the flow of information from the grid of one phase to the

Table 1. Grid independence study.

\begin{tabular}{lccccc}
\hline & \multicolumn{5}{c}{ Temperature at position (x,y) mm } \\
\cline { 2 - 6 } Nodes & $1.5,30$ & $1.5,60$ & $1.5,90$ & $1.5,120$ & $1.5,150$ \\
\hline 13294 & 392.67 & 377.62 & 365.89 & 359.00 & 355.66 \\
18494 & 395.92 & 379.70 & 367.16 & 359.78 & 356.17 \\
22722 & 394.53 & 376.74 & 363.83 & 356.47 & 352.97 \\
$\mathbf{2 5 0 8 8}$ & $\mathbf{3 9 6 . 3 2}$ & $\mathbf{3 7 9 . 8 3}$ & $\mathbf{3 6 7 . 5 7}$ & $\mathbf{3 6 0 . 0 4}$ & $\mathbf{3 5 6 . 6 7}$ \\
28457 & 396.86 & 381.05 & 368.64 & 360.96 & 356.65 \\
\hline
\end{tabular}

grid of the other phase as well as between fin (solid) and base (solid). The geometry of the model is shown in figure 3. A known heat flux is specified in the forward model (refer B.C.1 in figure 3) and Eqs. (2.1) to (2.5) is solved and the steady-state temperature distribution is obtained from the fin.

The governing equation with the appropriate boundary conditions is solved using the commercial ANSYS FLUENT 14.5 software. The fundamental equations of fluid flow are the continuity equation, momentum equations (Navier-Stokes equation) and energy equation. The properties of the materials considered for the conjugate heat transfer analysis are given in table 2 .

All the cases considered in the present study involve laminar, two-dimensional, steady convection. Boussinesq approximation is incorporated to account for the density change as a linear function of temperature for the buoyancy term in the momentum equation. Viscous heat dissipation and compressibility effects are not considered. Table 3 shows the boundary conditions used in the analysis. Location 2 of the solution domain is the solid-fluid interface for the flow of energy, momentum across the interface. Since the problem is conjugate heat transfer, the transfer of energy from aluminium base to steel fin is by conduction and that from the fin wall to the fluid medium as well as from aluminium base top to fluid medium is by convection. So, at the solid-fluid interfaces, the transfer of energy will be by convection. At location 8 , which is the solid-fluid interface, the information across the interface is prevented by providing insulation. Based on the above assumption, the governing equations can be written as follows. In the forward model, Eqs. (2.1) to (2.5) is solved by assuming the value for heat flux $q$.

Continuity

$$
\frac{\partial u}{\partial x}+\frac{\partial v}{\partial y}=0
$$

$\mathrm{X}$-momentum equation

$$
\mathrm{u} \frac{\partial u}{\partial x}+\mathrm{v} \frac{\partial u}{\partial y}=-\frac{1}{\rho} \frac{\partial p}{\partial x}+\vartheta\left(\frac{\partial^{2} u}{\partial x^{2}}+\frac{\partial^{2} u}{\partial y^{2}}\right)
$$

Y-momentum equation

$\mathrm{u} \frac{\partial v}{\partial x}+\mathrm{v} \frac{\partial v}{\partial y}=-\frac{1}{\rho} \frac{\partial p}{\partial y}+\vartheta\left(\frac{\partial^{2} v}{\partial x^{2}}+\frac{\partial^{2} v}{\partial y^{2}}\right)+g \beta\left(T-T_{\infty}\right)$

Energy equation (for fluid)

$$
\mathrm{u} \frac{\partial T}{\partial x}+\mathrm{v} \frac{\partial T}{\partial y}=\alpha\left(\frac{\partial^{2} T}{\partial x^{2}}+\frac{\partial^{2} T}{\partial y^{2}}\right)
$$

Energy equation (for solid)

$$
\mathrm{k}_{\mathrm{s}}\left(\frac{\partial^{2} T}{\partial x^{2}}+\frac{\partial^{2} T}{\partial y^{2}}\right)=0
$$




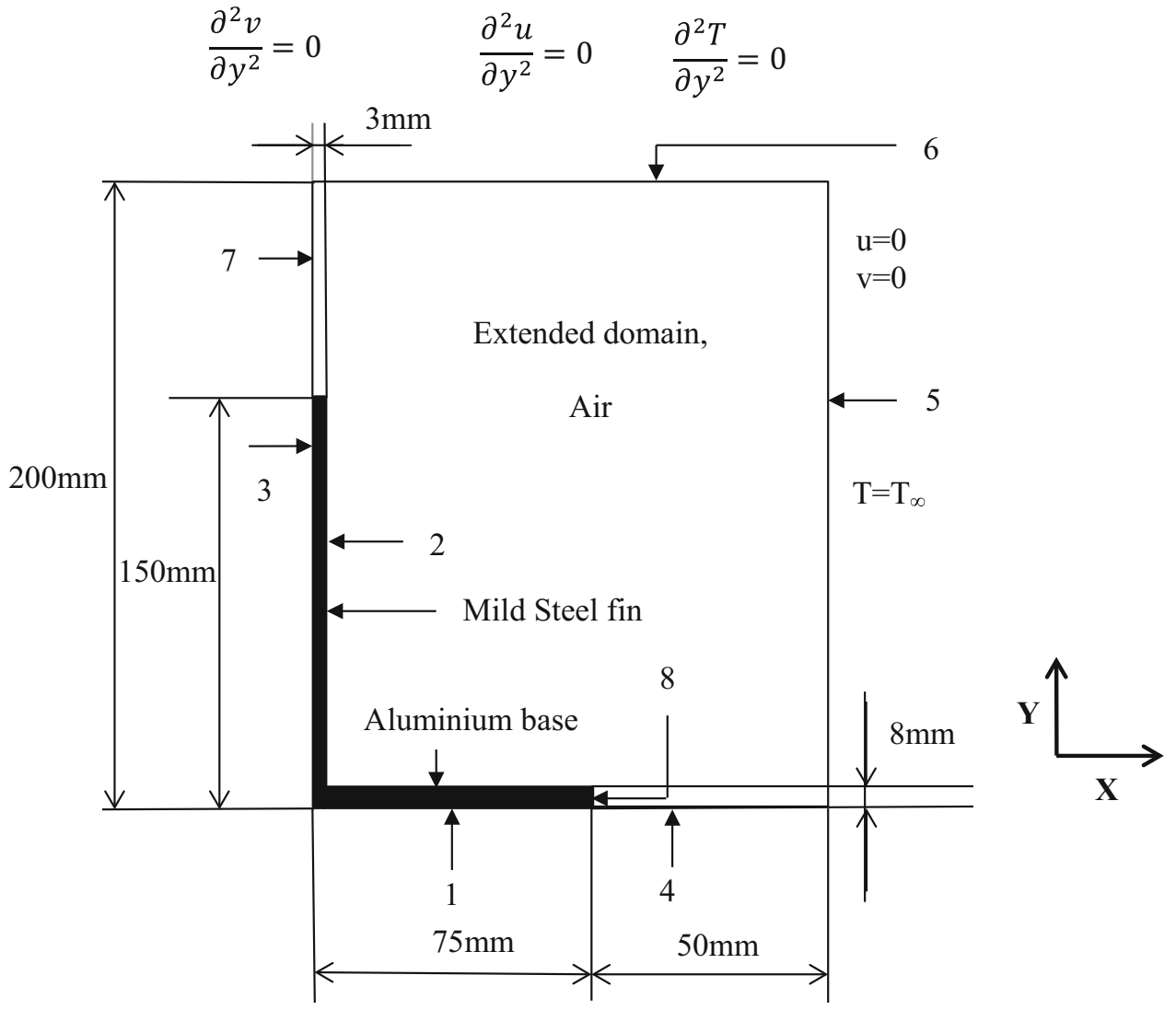

Figure 3. Schematic representation of the geometry model and boundary conditions.

Table 2. Properties of the materials used in simulation.

\begin{tabular}{lcc}
\hline Properties & Mild steel & Aluminium \\
\hline Density $\left(\mathrm{kg} / \mathrm{m}^{3}\right)$ & 8030 & 2719 \\
Specific heat $(\mathrm{J} / \mathrm{kg} \mathrm{K})$ & 502.48 & 871 \\
Thermal conductivity $(\mathrm{W} / \mathrm{m} \mathrm{K})$ & 37 & 202.4 \\
\hline
\end{tabular}

Table 3. Types of boundary conditions for analysis.

\begin{tabular}{cc}
\hline Boundary & Type of boundary condition \\
\hline 1 & $q=-k \frac{\partial T}{\partial x}$ \\
2 & Solid-fluid interface \\
3 & $\frac{\partial T}{\partial x}=0$ \\
4 & Pressure outlet \\
5 & Pressure outlet \\
6 & Pressure outlet \\
7 & $\frac{\partial T}{\partial x}=0$ \\
8 & Solid-fluid interface \\
\hline
\end{tabular}

\subsection{Forward model validation}

The forward model is validated against the known exact solution, which is available for the constant area fin (convecting at the tip) subjected to steady-state heat transfer. The governing differential equation of the fin under steady state is given by

$$
\frac{\partial^{2} \theta}{\partial X^{2}}-(m L)^{2} \theta=0
$$

Where

$$
\theta=\frac{T-T_{\infty}}{T_{o}-T_{\infty}}, \mathrm{X}=\frac{x}{L}
$$

The boundary conditions are

$$
\begin{aligned}
& \mathrm{x}=0, \theta(0)=1 \\
& \mathrm{x}=\mathrm{L}, \mathrm{h} \theta(\mathrm{L})=-\mathrm{k} \frac{d \theta}{d x}
\end{aligned}
$$

The temperature distribution is obtained as [26]

$$
\theta=\frac{\cosh m(L-x)+\left(\frac{h}{m k}\right) \sinh m(L-x)}{\cosh m L+\left(\frac{h}{m k}\right) \sinh m L}
$$

The temperature distribution of the forward model and the analytical solution are compared and reported in table 4 . The maximum error is found to be $0.37 \%$. Thus, the validation of 
Table 4. Comparison between temperatures obtained from CFD and analytical solution.

\begin{tabular}{cccc}
\hline $\begin{array}{l}\text { Position, } x \\
(\mathrm{~m})\end{array}$ & $\begin{array}{c}\text { Simulated } \\
\text { temperature }(\mathrm{K})\end{array}$ & $\begin{array}{c}\text { Analytical } \\
\text { temperature }(\mathrm{K})\end{array}$ & $\begin{array}{c}\text { Error } \\
(\%)\end{array}$ \\
\hline 0.03 & 342.32 & 341.43 & 0.25 \\
0.06 & 335.18 & 335.93 & 0.22 \\
0.09 & 331.28 & 332.12 & 0.25 \\
0.12 & 328.64 & 329.87 & 0.37 \\
\hline
\end{tabular}

the forward model ensures the reliability and accuracy of the computational model for further simulations.

\subsection{Fast forward model - ANN}

Equations (2.1) to (2.5) are solved in FLUENT to obtain the temperature distribution of the fin. One typical numerical solution takes $20 \mathrm{~min}$ for convergence. A convergence criteria of $1 \times 10^{-6}$ is fixed for the numerical analysis. As specified in figure 2 , the estimation process is an iterative technique and the numerical solution (forward model) should be executed for every sample during the iterative process. In this manner, the computational cost involved in solving the forward model is high and time consuming. Hence, one can avoid or reduce the computation cost by replacing the existing forward model with a simple or fast forward model based on new techniques.

The fast forward model used in the present work is ANN. This is a computational tool used to find out relation between large number of input and output data. ANN can be basically characterised as multi-layer perceptron (MLP), radial basis function network (RBF), adaptive linear network (ADALINE) and more. Neural network resembles biological nervous systems. This system approximates functions according to the numerous known inputs and outputs provided to the network. Inspired by the biological neural network, ANNs are massively parallel computing system consisting of an extremely large number of simple processors with many interconnections. It is used to estimate the relationship between a large number of input and output parameters and finally predicts the output for the input given within the range used during creation of the network. This is done by interconnected neurons and processing elements called nodes. Each node is associated with some weight which is used to determine the strength of the signal input to find the output as weighted sum of these different inputs. Training is adjustment of these weights for each neuron to approximate the function with least error possibilities. In general, 70-75\% of the data are considered for training and the remaining used for testing purpose. Training is accomplished by using a set of inputs for which the output is known. A supervised learning algorithm called the back propagation algorithm is used in the present work. The Levenberg-Marquardt algorithm is used as the training function to arrive at the network output at a faster rate. In an MLP network, the nodes at each layer are fully connected to the nodes in the next one. Each node in one layer connects with a specific weight to every node in the next layer. The network has three interfaces, namely input layer, hidden layer and output layer. The signal flows from input layer to the destined output layer with variations occurring in hidden middle layer. Figure 4 shows the depiction of a typical neural network model.

Training is accomplished using a set of network inputs for which the desired outputs are known. The selection of the number of hidden neurons is based on the values of some of the common performance metrics used [12], as follows. This is termed as neuron independence study as shown in table 5. Based on this study, eight neurons have been considered for training the network.

Mean Relative Error

$$
\mathrm{MRE}=\frac{1}{\mathrm{~N}} \sum_{\mathrm{i}=1}^{\mathrm{N}} \frac{\left|\mathrm{t}_{\text {actual }, \mathrm{i}}-\mathrm{t}_{\text {network }, \mathrm{i}}\right|}{\left|\mathrm{t}_{\text {actual }, \mathrm{i}}\right|}
$$

Correlation Coefficient

$$
\mathrm{R}^{2}=1-\frac{\sum_{i=1}^{\mathrm{N}}\left(\mathrm{t}_{\text {actual }, \mathrm{i}}-\mathrm{t}_{\text {network }, \mathrm{i}}\right)^{2}}{\sum_{\mathrm{i}=1}^{\mathrm{N}}\left(\mathrm{t}_{\text {actual }, \mathrm{i}}^{2}\right)}
$$

\section{Inverse model}

\subsection{Bayesian inference}

Bayesian inference is used as a framework to minimise the objective function. The inverse model is based on the

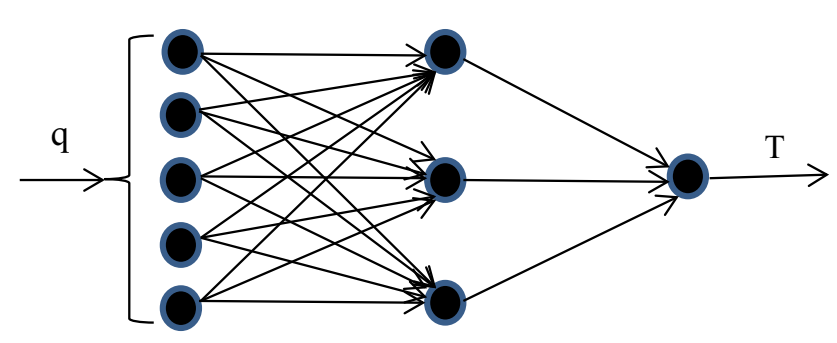

Input layer

Hidden layer

Output layer

Figure 4. Layout of neural network.

Table 5. Neuron independence study.

\begin{tabular}{cccc}
\hline No. of neurons & MRE & \multicolumn{1}{c}{$\mathrm{R}^{2}$} & $\mathrm{R}_{\text {test }}$ \\
\hline 4 & 0.00664 & 0.99993443 & 0.997818 \\
6 & 0.00700 & 0.999922362 & 0.999949 \\
$\mathbf{8}$ & $\mathbf{0 . 0 0 6 0 8}$ & $\mathbf{0 . 9 9 9 9 4 1 2 8 8}$ & $\mathbf{0 . 9 9 9 9 4 9}$ \\
10 & 0.00656 & 0.999938161 & 0.997362 \\
13 & 0.00617 & 0.999940957 & 0.997676 \\
\hline
\end{tabular}


Bayes' theorem to relate the experimental/surrogate data $Y$ and the parameter $x$ is as follows:

$$
\mathrm{P}(\mathrm{x} / \mathrm{Y})=\frac{\mathrm{P}(\mathrm{Y} / \mathrm{x}) \mathrm{P}(\mathrm{x})}{\mathrm{p}(\mathrm{Y})}
$$

where $\mathrm{P}(x / Y)$ is the posterior probability density function (PPDF); $\mathrm{P}(Y / x)$ is the likelihood function or the forward model; $\mathrm{P}(x)$ is the prior density function; and $\mathrm{P}(Y)$ is the normalising constant.

In Eq. (3.1), ' $x$ ' is often termed as hypothesis [4]. Bayesian inference forms a powerful technique for estimation, hypothesis testing, decision making and selection of the model. Given the observations, the posterior probability of a hypothesis is proportional to the product of its likelihood and prior probability. The likelihood $\mathrm{P}(Y / x)$ provides the probability of obtaining the data vector $(Y)$ for a given parameter vector $x$ (direct problem). $\mathrm{P}(x)$ gives any prior knowledge about the estimators. Prior can be of three types [7, 27]: Gaussian prior, log-normal prior and uniform prior. [8]

The Bayes' theorem can also be stated as in ref.

$$
\begin{gathered}
\mathrm{P}_{\text {post }}(q / T)=\frac{P_{f}(T / q) P_{p r}(q)}{\int P_{f}(T /(q) \operatorname{Ppr}(q) d q} \\
P(T / q)=\left(2 \pi \sigma^{2}\right)^{\frac{-n}{2}} \\
. \exp \left\{\frac{-1}{2 \sigma^{2}}\left[T_{\text {meas }}-T_{\text {sim }}(q)\right]^{T}\left[T_{\text {meas }}-T_{\text {sim }}(q)\right]\right\} \\
P(q)=\left(2 \pi \sigma^{2}\right)^{-0.5} \cdot \exp \left\{\frac{-1}{2 \sigma_{p}^{2}}\left(q-\mu_{p}\right)^{2}\right\} \\
\mathrm{P}_{\text {post }}(q / T)=\left(2 \pi \sigma^{2}\right)^{\frac{-n}{2}} \exp _{\left.\frac{-1}{2 \sigma^{2}}\left[T_{\text {meas }}-T_{\text {sim }}(q)\right]^{T}\left[T_{\text {meas }}-T_{\text {sim }}(q)\right]\right\}}\left(2 \pi \sigma^{2}\right)^{-0.5} \cdot \exp \left\{\frac{-1}{2 \sigma_{p}^{2}}\left(q-\mu_{p}\right)^{2}\right\}
\end{gathered}
$$

Equation (3.3) provides the expression for the likelihood function, which is, in other terms, also called as the forward model. The uncertainty $\sigma$ is due to the measurement errors and the error in the forward model. The PPDF is expressed as the product of $P(T / q)$ and $P(q)$. For a uniform prior, the PPDF now becomes $P(T / q)$; that is, the posterior probability density is equivalent to the likelihood function. In Eq. (3.3), $n$ represents the total number of temperatures measured in the experiment. A frequently employed prior is the Gaussian prior, which is given by Eq. (3.4). When the likelihood function follows a normal distribution, the mean value represents the estimate of the unknown parameter. The mean expression is given as

$$
\operatorname{Mean}\left(x_{r e t}\right)=\frac{\sum_{i} q_{i} e\left[-\frac{1}{2}\left(x_{i}^{2}+\left(\left(q-\mu_{p}\right)^{2} / \sigma_{p}^{2}\right)\right)\right]}{\sum_{i} e\left[-\frac{1}{2}\left(x_{i}^{2}+\left(\left(q-\mu_{p}\right)^{2} / \sigma_{p}^{2}\right)\right)\right]}
$$

Where $\sum_{i}$ spans all the points in the database. The estimate can also be reported in terms of maximum of the PPDF and it is given as

$$
\mathrm{MAP}=\arg \max [P(q \mid T)]
$$

\subsection{Metropolis-Hastings algorithm}

The sampling algorithm used in this work is Metropolis-Hastings algorithm [8]. It has been found in the literature that the $\mathrm{MH}$ algorithm is very powerful and the problem of being trapped in to local minima/maxima is avoided. The procedure for the algorithm is given as

a. Initialise $x_{1}$

b. for $i=1 \ldots, \mathrm{M}$

1. Draw a sample $\mathrm{u} \sim \mathrm{U}(0,1)$, that is, from a uniform distribution between 0 and 1 .

2. Draw a sample $x^{*} \sim q\left(x^{*} / x^{i}\right)$

3. If $\mathrm{u}<\mathrm{A}\left(\mathrm{x}^{*}, \mathrm{x}^{\mathrm{i}}\right), \mathrm{x}_{\mathrm{i}+1}=\mathrm{x}^{*}$

4. Else go to step 2 with $x_{i+1}=x^{i}$

Acceptance function, $\quad \mathrm{A}\left(\mathrm{x}^{*}, \mathrm{x}^{\mathrm{i}}\right)=\min$ $\left(1,\left(\frac{P\left(x_{*}\right) \cdot q\left(\frac{x_{i}}{x_{*}}\right)}{P\left(x_{i}\right) \cdot q\left(\frac{x_{*}}{x_{i}}\right)}\right)\right)$.

\section{Sensitivity analysis}

It is important to carry out the sensitivity analysis to determine the behaviour and characteristics of the unknown parameter before performing the estimation process. The sensitivity study is carried out to know the behaviour of the estimated temperature with the changes in the parameter. The sensitivity analysis is the Jacobian matrix that investigates the behaviour between the inputs and the outputs. The objective of SA is to identify how the variability in an output quantity of interest $\left(T_{i}\right)$ is connected to an input $\left(P_{j}\right)$ in the model; the result is a sensitivity derivative $\left(\frac{\partial T_{i}}{\partial P_{j}}\right)$. Before addressing the estimation of the unknown parameters, the behaviour of the determinant of the information matrix $\mathbf{J}^{\mathbf{T}} \mathbf{J}[2,16]$ is determined. The information matrix is also called as sensitivity matrix $\mathbf{J}$ and the entries of the matrix $J_{i j}$ are 
called sensitivity coefficients. The sensitivity matrix [2] is defined as

$$
J(P)=\left[\frac{\partial T^{T}(P)}{\partial P}\right]^{T}=\left[\begin{array}{ccccc}
\frac{\partial T_{1}}{\partial P_{1}} & \frac{\partial T_{1}}{\partial P_{2}} & \frac{\partial T_{1}}{\partial P_{3}} & \cdots & \frac{\partial T_{1}}{\partial P_{N}} \\
\frac{\partial T_{2}}{\partial P_{1}} & \frac{\partial T_{2}}{\partial P_{2}} & \frac{\partial T_{2}}{\partial P_{3}} & \cdots & \frac{\partial T_{2}}{\partial P_{N}} \\
\vdots & \vdots & \vdots & & \vdots \\
\frac{\partial T_{I}}{\partial P_{1}} & \frac{\partial T_{I}}{\partial P_{2}} & \frac{\partial T_{I}}{\partial P_{3}} & \cdots & \frac{\partial T_{1}}{\partial P_{N}}
\end{array}\right]
$$

The present work involves Navier-Stokes equations and it is not easy to obtain the Jacobian matrix. As a representative case, the steady-state temperature using Green's function from ref. [16] is considered for a fin with high thermal conductivity and an attempt has been made to calculate the sensitivity coefficient. The steady-state temperature is given as

$$
\begin{gathered}
T(x, t)-T_{\infty}=\frac{q L}{k} \frac{\left(e^{-m x}-e^{-m(2 L-x)}\right)}{m L\left(1+e^{-2 m L}\right)} \\
\frac{\partial \mathrm{T}_{\mathrm{i}}}{\partial \mathrm{q}}=\left(\frac{\mathrm{e}^{-\mathrm{mx}_{\mathrm{i}}}-\mathrm{e}^{-\mathrm{m}\left(2 \mathrm{~L}-\mathrm{x}_{\mathrm{i}}\right)}}{\mathrm{mL}\left(1+\mathrm{e}^{-2 \mathrm{~mL}}\right)}\right) \frac{\mathrm{L}}{\mathrm{k}}
\end{gathered}
$$

The sensitivity coefficient with respect to an unknown parameter $\mathrm{P}_{\mathrm{j}}$ ( $\mathrm{q}$ in this case) is determined by differentiating the solution with respect to $P_{j}$. The plot of sensitivity coefficient is shown in figure 5 . When the determinant of $\mathbf{J}^{\mathrm{T}} \mathbf{J} \approx 0$, then the inverse problem is ill conditioned. In such conditions, prior information is essential for estimation of the parameter. Therefore, it is desirable to have linearly independent sensitivity coefficients $\mathrm{J}_{\mathrm{ij}}$ with large magnitudes, such that the inverse problem is not very sensitive to measurement errors and accurate estimates of the parameters can be obtained. From figure 5, it is seen that the trend of the graph is decreasing and one can come to a conclusion that the estimation of heat flux is possible when the temperature sensor is closer to the base of the fin because of the

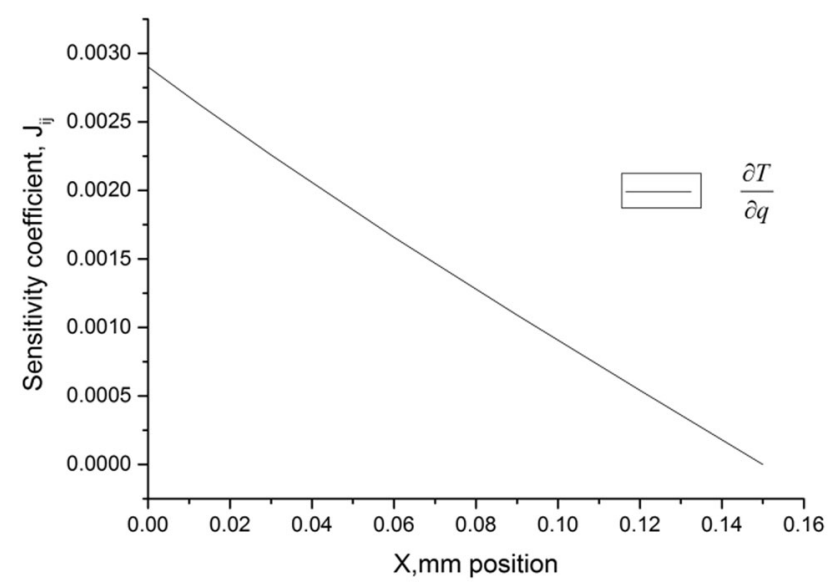

Figure 5. Plot of sensitivity coefficients along with positions. fact that sensitivity coefficient $\mathrm{J}_{\mathrm{ij}}$ tends to zero and the information is no more useful to estimate the heat flux.

\section{Results and discussion}

There are various iterative methods available for the estimation of unknown parameters in inverse heat transfer problem. In the present work, deterministic gradient-based methods cannot be used because of the complexity involved in obtaining the Jacobian matrix. A stochastic-based approach is used for the present conjugate heat transfer problem, which helps eliminate the tedious calculation of Jacobian matrix.

\subsection{Forward solution from numerical model (CFD)}

The objective of the work is to show the validity of the Bayesian framework in estimating heat flux with uniform prior and normal prior information about the unknown heat flux for the perturbed surrogated data. The forward model consists of a numerical model and the geometry is modelled and meshed using ANSYS 14.5 with the selected grid size. The numerical analysis is carried out in the meshed region with the specified boundary conditions and convergence criteria of $1 \times 10^{-6}$. The heat flux applied at the base is varied from 1100 to $2300 \mathrm{~W} / \mathrm{m}^{2}$. The selection of heat flux range confirms that the Rayleigh number does not exceed the critical value and the flow remains laminar. Steady-state heat transfer simulations are carried out for different values of heat flux and the corresponding temperature data are obtained. Figures 6 and 7 show the temperature distribution and velocity contour for $1200 \mathrm{~W} / \mathrm{m}^{2}$ respectively.
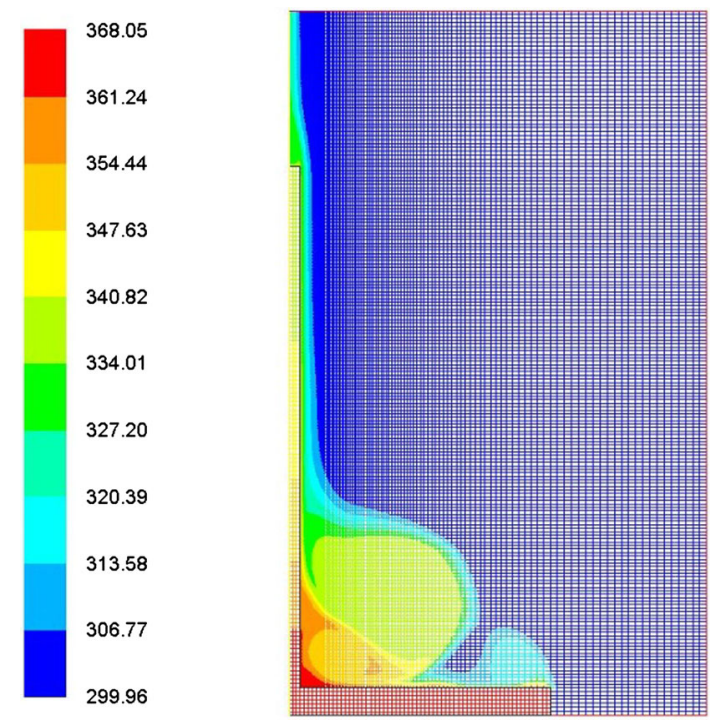

Figure 6. Temperature plot along the length of the fin at heat flux $1200 \mathrm{~W} / \mathrm{m}^{2}$ from CFD simulation. 


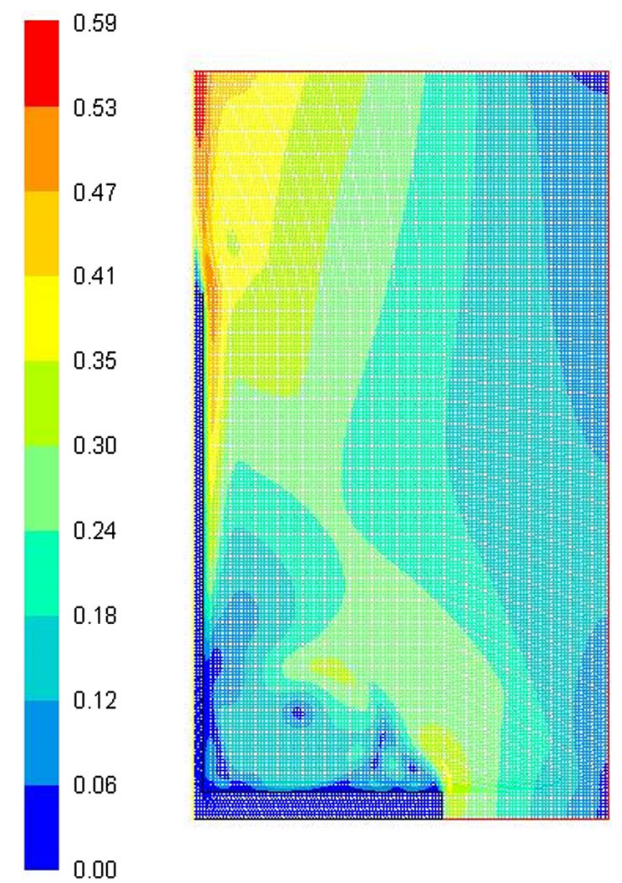

Figure 7. Velocity plot at heat flux $1200 \mathrm{~W} / \mathrm{m}^{2}$ from CFD simulation.

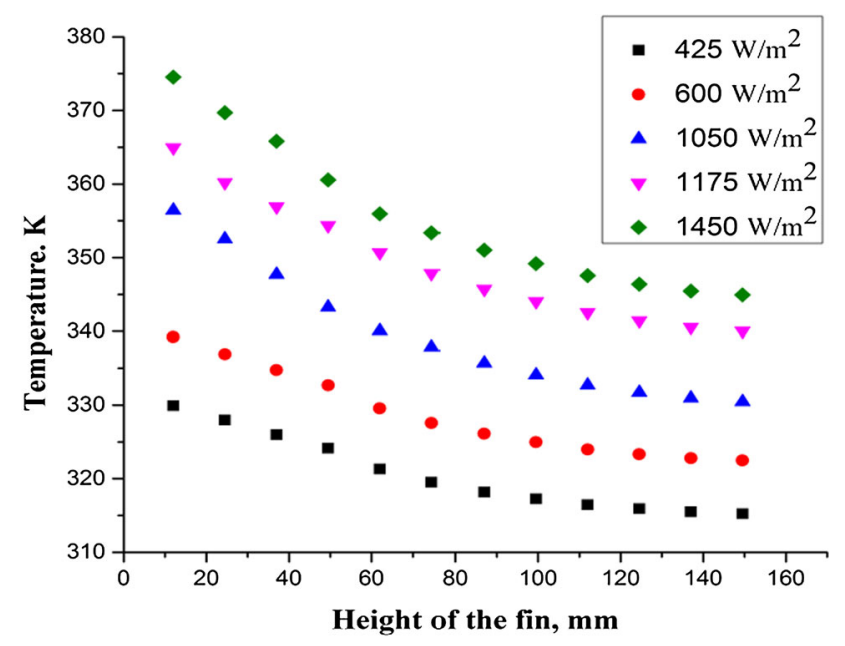

Figure 8. Variation in temperature with position for different heat flux.

Simulations are carried out for different heat flux and the temperature distribution is obtained for each case. Figure 8 shows the temperature distribution along the length of fin for various heat flux values.

\subsection{Forward solution from numerical model $(A N N)$}

As mentioned previously, numerical simulations are now replaced with ANN for different heat flux values that act as
Table 6. Comparison between temperatures obtained from CFD and ANN.

\begin{tabular}{rccc}
\hline $\begin{array}{l}\text { Heat flux, } \\
\mathrm{q}, \mathrm{W} / \mathrm{m}^{2}\end{array}$ & $\begin{array}{c}\text { Simulation } \\
\text { temperature, } \mathrm{K} \\
\text { (CFD) }\end{array}$ & $\begin{array}{c}\text { Forward model } \\
\text { temperature, K } \\
\text { (ANN) }\end{array}$ & $\begin{array}{c}\text { Error } \\
(\%)\end{array}$ \\
\hline 930 & 339.36 & 338.20 & 0.34 \\
1050 & 340.04 & 343.11 & 0.90 \\
1280 & 349.49 & 351.54 & 0.58 \\
1450 & 355.93 & 356.12 & 0.05 \\
\hline
\end{tabular}

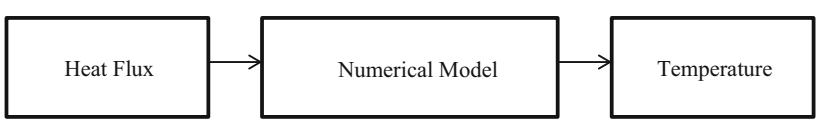

Figure 9. Schematic representation of surrogated temperature.

a fast forward model for further simulations. Table 6 shows the comparison between CFD and ANN temperature at a location $(x, y),(1.5,62) \mathrm{mm}$. It has been observed that a maximum error of $0.9 \%$ is noticed, which indicates that the forward model (ANN) used in this work predicts values very close to the simulated temperature (CFD).

\subsection{Estimation of unknown heat flux}

In this section, an attempt is made for the estimation of heat flux from the fin, for the known experimental or surrogated temperature. The unknown parameter is estimated with and without subjective prior. Initially, the unknown heat flux is estimated without considering the prior information. This work considers the surrogated temperature data instead of experimental temperature. In this case, the PPDF is directly equal to the likelihood function. Surrogated data added with noise mimics the experimental data. Figure 9 shows the information about the surrogated data. An assumed heat flux from the specified range is given as input to the forward model to obtain the surrogated temperature. Now, the problem turns out to be single-parameter estimation where the temperature is known and heat flux is assumed to be unknown.

Furthermore, the error between ANN and the surrogated temperature is minimised using Bayesian methodology and the unknown heat flux is estimated. The forward model measurement error is assumed to be 5\%. With the help of MH-MCMC algorithm, the heat flux is estimated for various values of surrogated temperature. The effect of number of samples on estimation is discussed in table 7. It is observed that as the number of samples is increased, the changes in the point estimates are negligible. Hence, 5000 samples are considered for faster computational rate. 
Table 7. Effect of sample size on Bayesian retrieval of heat flux.

\begin{tabular}{lcccc}
\hline & & \multicolumn{2}{c}{$\begin{array}{c}\text { Bayesian retrieved values } \\
\text { for } \mathrm{q}\left(\mathrm{W} / \mathrm{m}^{2}\right)\end{array}$} \\
\cline { 3 - 5 } $\begin{array}{l}\text { Heat flux, q } \\
\mathrm{W} / \mathrm{m}^{2}\end{array}$ & $\begin{array}{c}\text { Number of } \\
\text { samples }\end{array}$ & Mean & MAP & $\mathrm{SD}$ \\
\hline 1200 & 5000 & 1200.27 & 1200.46 & 0.314 \\
& 10000 & 1200.36 & 1200.45 & 0.312 \\
& 15000 & 1200.26 & 1198.90 & 0.313 \\
& 20000 & 1200.71 & 1198.83 & 0.316 \\
\hline
\end{tabular}

The PPDF of heat flux $1200 \mathrm{~W} / \mathrm{m}^{2}$ plotted against different samples is shown in figure 10. As seen from table 7 , the standard deviation of the estimated heat flux is very less and the same is reflected in the PPDF plot. The mean and the MAP of the estimated heat flux reported in table 7 for different samples do not vary significantly, and both predict closer to the known heat flux value. This again proves the robustness of the Bayesian framework.

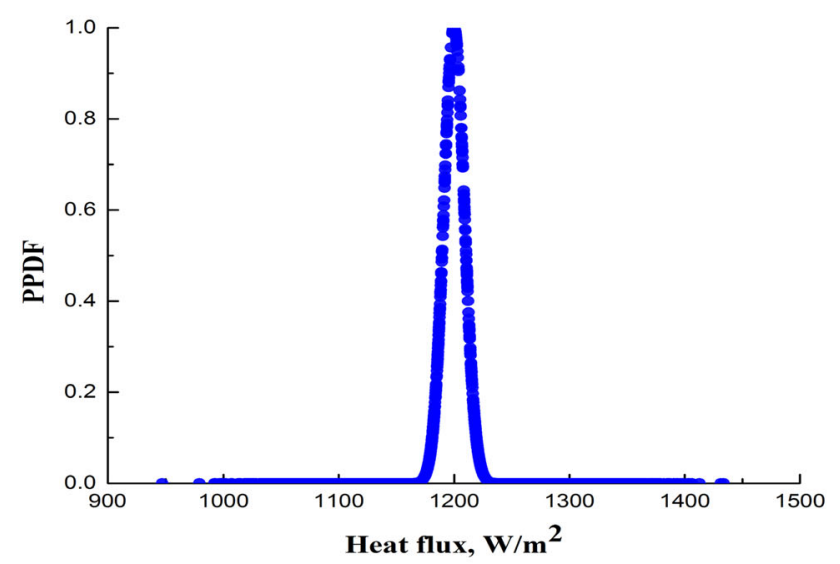

(a)

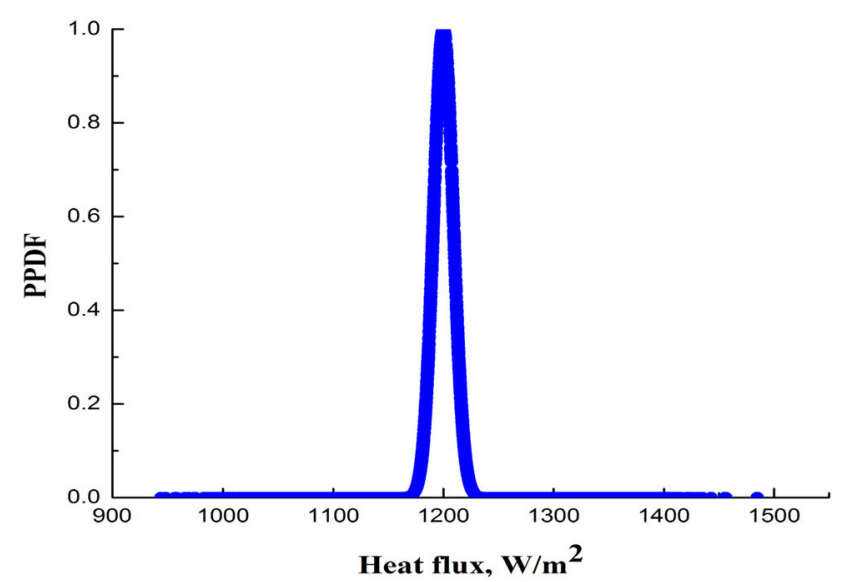

(c)
Figure 11 represents the frequency distribution of the heat flux and is clearly seen that the samples oscillate with respect to the mean $1200 \mathrm{~W} / \mathrm{m}^{2}$. In case of uniform prior, the PPDF is equal to the likelihood function and Eq. (3.3) is used for estimating heat flux. When a Gaussian prior is incorporated, the PPDF now becomes Eq. (3.5). Gaussian prior is incorporated to capture the prior knowledge of the parameter. The expression for the Gaussian prior is given by Eq. (3.4).

From table 8 , it is seen that the assumed value of heat flux used to obtain surrogated temperature and the heat flux estimated using MH-MCMC algorithm agrees very well for both the prior models considered. The estimated values of heat flux is reported as mean, MAP and standard deviation in which the uncertainty associated with the estimated parameters can directly be obtained using the Bayesian framework. There is no significant difference between the heat flux values estimated by using uniform prior and normal prior because the surrogated temperature contains no noise. Table 8 also shows the comparison between the

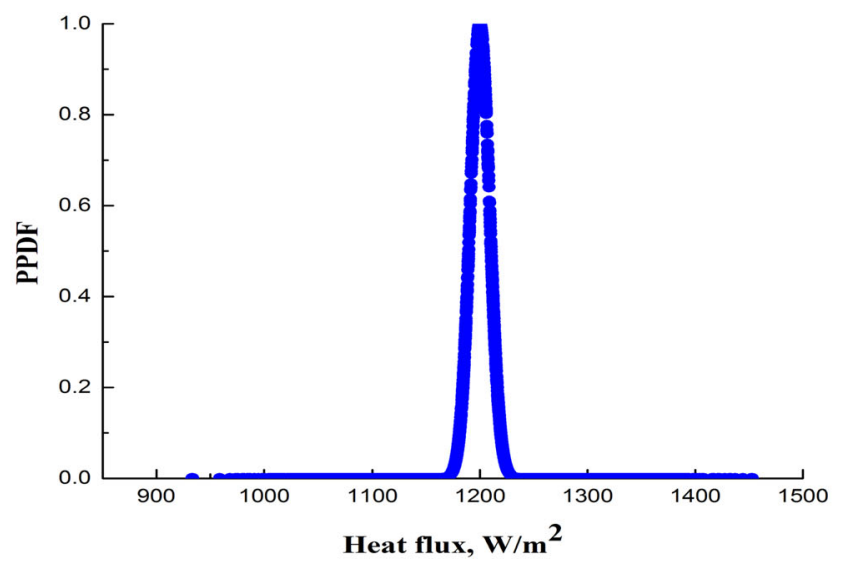

(b)

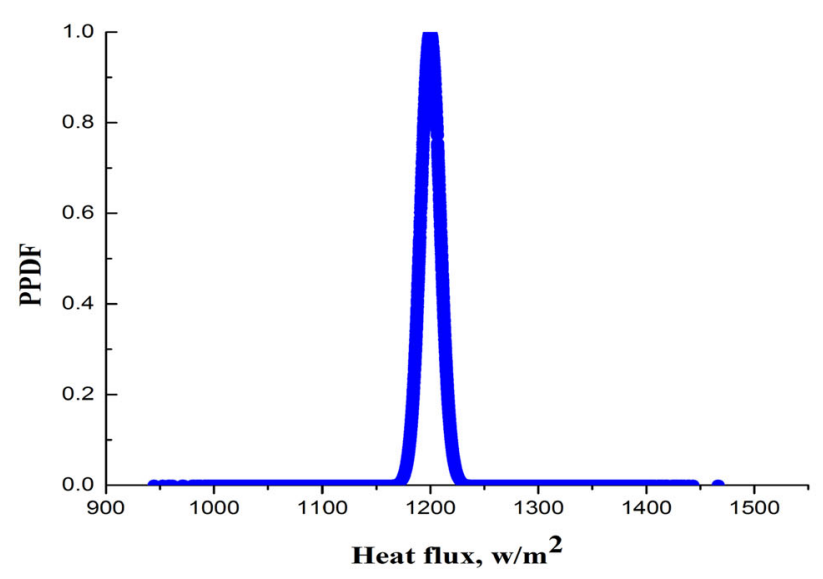

(d)

Figure 10. PPDF plot for $(\mathbf{a})=5000$ samples, $(\mathbf{b})=10000$ samples, $(\mathbf{c})=15000$ samples, $(\mathbf{d})=20000$ samples. 


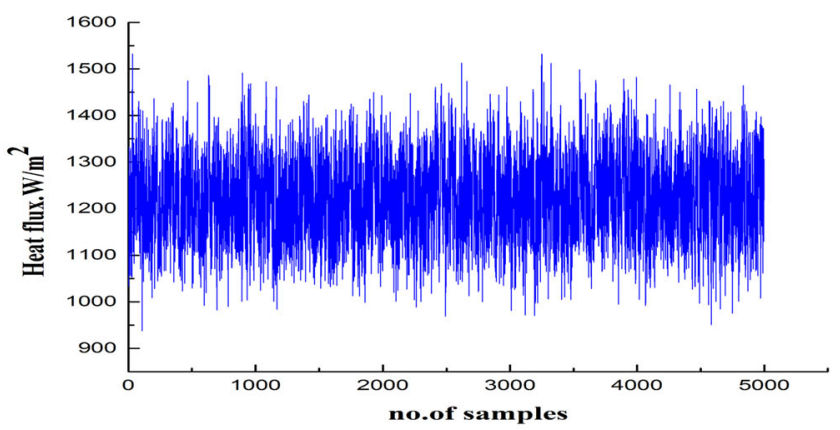

(a)

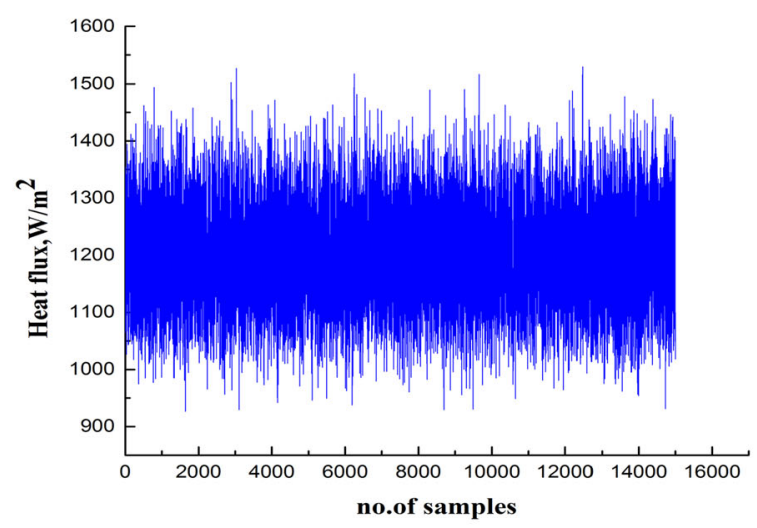

(c)

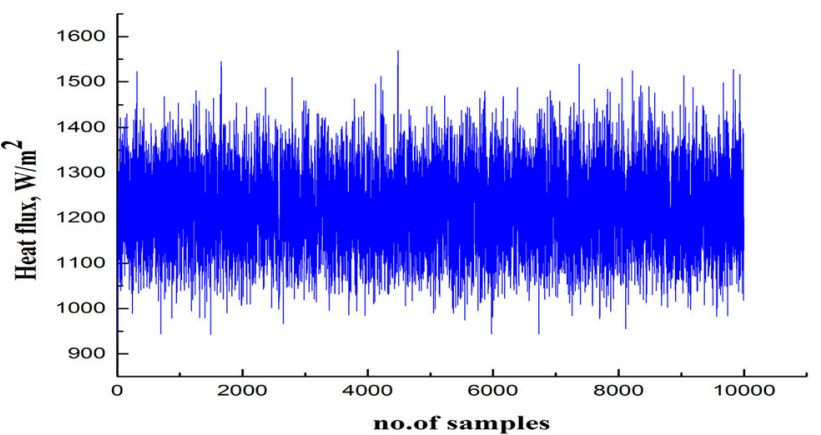

(b)

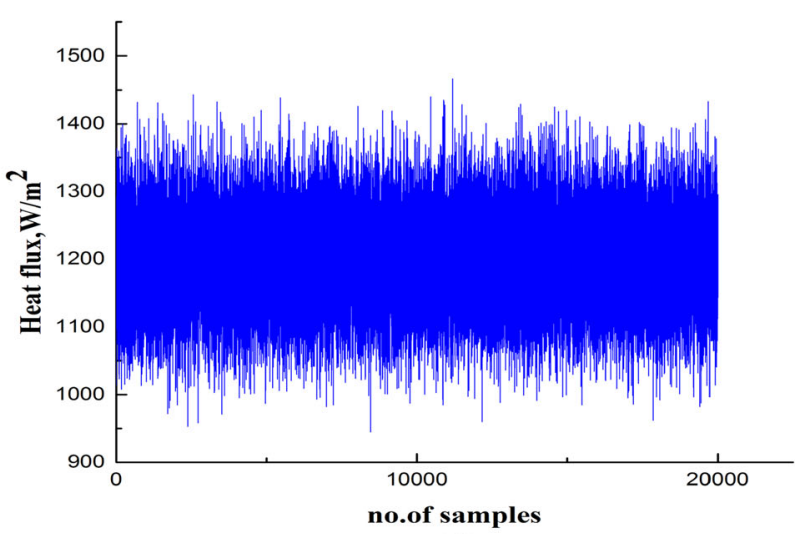

(d)

Figure 11. Frequency histogram data of heat flux $1200 \mathrm{~W} / \mathrm{m}^{2}$ for $(\mathbf{a})=5000$ samples, $(\mathbf{b})=10000$, samples, $(\mathbf{c})=15000$ samples, $(\mathbf{d})=$ 20000 samples.

Table 8. Retrieved values of heat flux using MH-MCMC with prior and without prior.

\begin{tabular}{|c|c|c|c|c|c|c|c|}
\hline \multirow[b]{2}{*}{ Heat flux } & \multirow[b]{2}{*}{ Prior model } & \multicolumn{3}{|c|}{ Number of samples $=5000$} & \multicolumn{3}{|c|}{ Number of samples $=10000$} \\
\hline & & Mean & MAP & SD & Mean & MAP & SD \\
\hline \multirow[t]{2}{*}{930} & Uniform & 929.76 & 929.77 & 0.310 & 929.93 & 929.25 & 0.300 \\
\hline & Normal & 929.41 & 929.61 & 0.300 & 929.31 & 929.46 & 0.290 \\
\hline \multirow[t]{2}{*}{1050} & Uniform & 1050.25 & 1050.64 & 0.290 & 1050.11 & 1048.86 & 0.290 \\
\hline & Normal & 1047.90 & 1048.20 & 0.256 & 1048.10 & 1048.30 & 0.246 \\
\hline \multirow[t]{2}{*}{1450} & Uniform & 1451.47 & 1448.67 & 0.430 & 1451.26 & 1448.92 & 0.450 \\
\hline & Normal & 1447.00 & 1447.00 & 0.426 & 1447.10 & 1447.80 & 0.381 \\
\hline
\end{tabular}

Table 9. Estimation of heat flux for perturbed data at different noise levels.

\begin{tabular}{|c|c|c|c|c|c|c|c|}
\hline \multirow[b]{2}{*}{ Heat flux, $\mathrm{W} / \mathrm{m}^{2}$} & \multirow[b]{2}{*}{ Prior model } & \multicolumn{3}{|c|}{ Noise level $=0.1 \mathrm{~K}$} & \multicolumn{3}{|c|}{ Noise level $=0.5 \mathrm{~K}$} \\
\hline & & Mean & MAP & SD & Mean & MAP & SD \\
\hline \multirow[t]{2}{*}{930} & Uniform & 932.17 & 932.46 & 0.312 & 942.28 & 942.09 & 0.307 \\
\hline & Normal & 930.16 & 930.86 & 0.284 & 938.72 & 939.85 & 0.301 \\
\hline \multirow[t]{2}{*}{1050} & Uniform & 1052.6 & 1052.4 & 0.291 & 1062.8 & 1062.5 & 0.288 \\
\hline & Normal & 1050.4 & 1050.4 & 0.245 & 1059.2 & 1059.8 & 0.257 \\
\hline \multirow[t]{2}{*}{1450} & Uniform & 1455.0 & 1454.0 & 0.461 & 1473.4 & 1473.6 & 0.472 \\
\hline & Normal & 1451.5 & 1451.0 & 0.369 & 1466.7 & 1467.3 & 0.392 \\
\hline
\end{tabular}




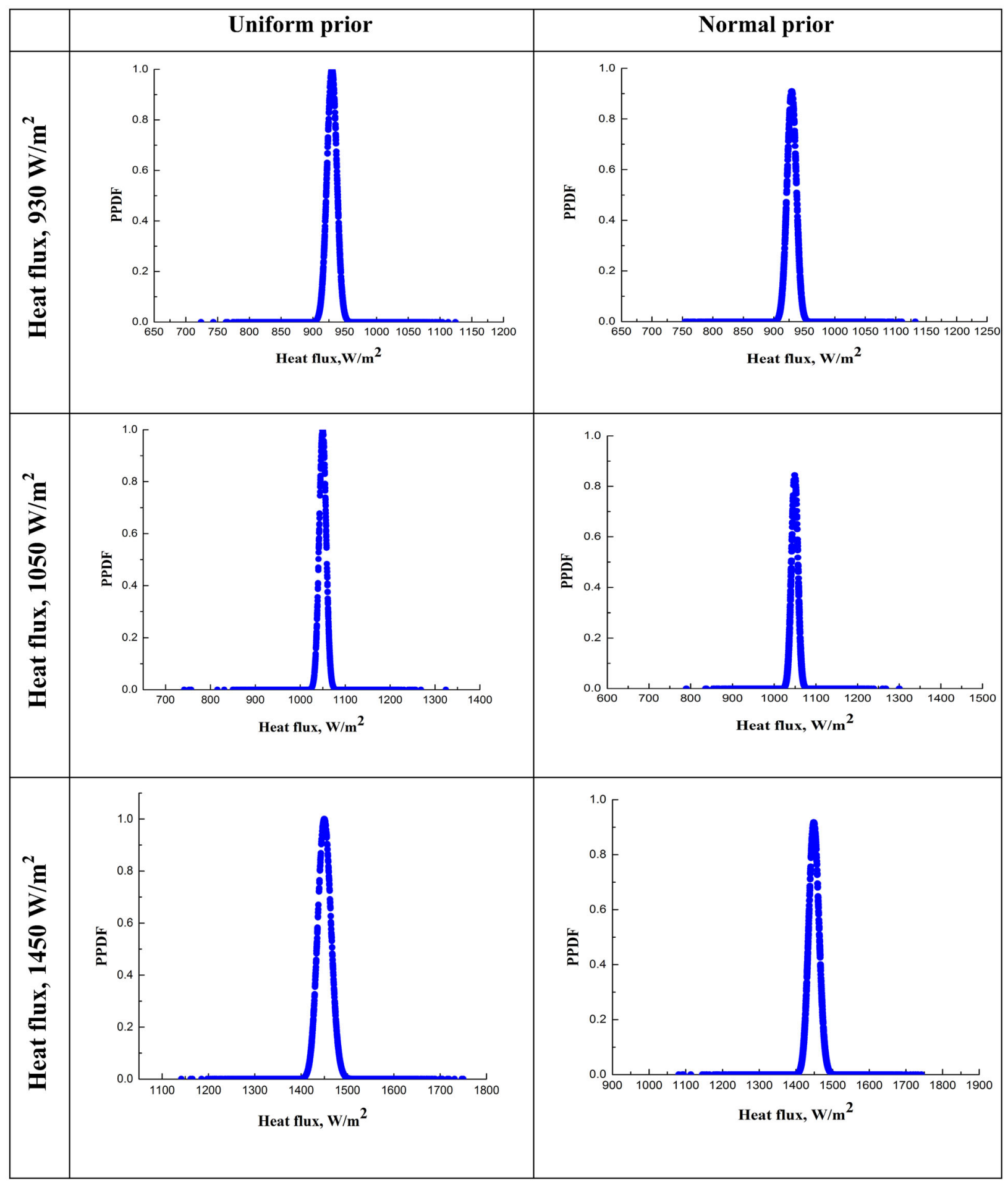

Figure 12. PPDF for different heat flux values with uniform and normal prior.

number of samples considered for the estimation process. To study the importance of prior information for a conjugate heat transfer problem, different priors are specified in Eq. (3.5) and the unknown heat flux is estimated. However, the standard deviation of prior plays important role during the estimation process. For this purpose, the standard deviation $\sigma_{\mathrm{p}}$ is assumed to be $5 \%$ and $2 \%$ and the corresponding results are shown in tables 8 and 9 respectively. 


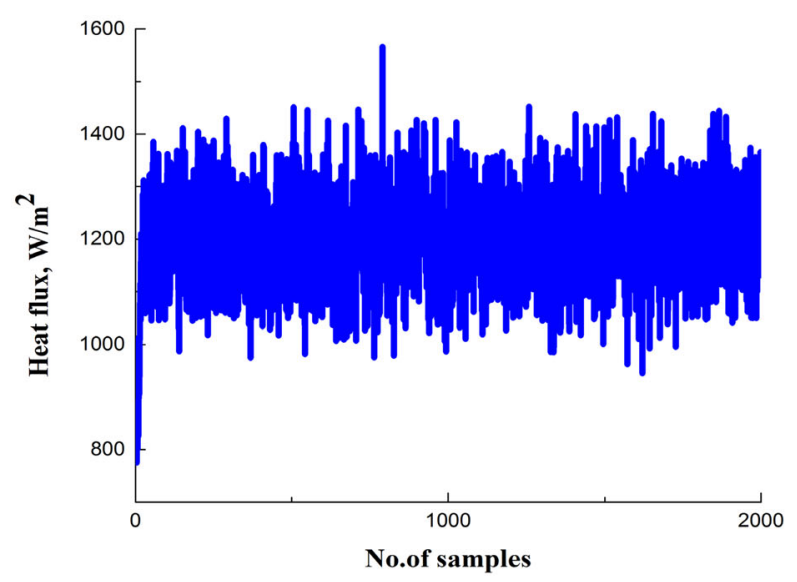

(a)

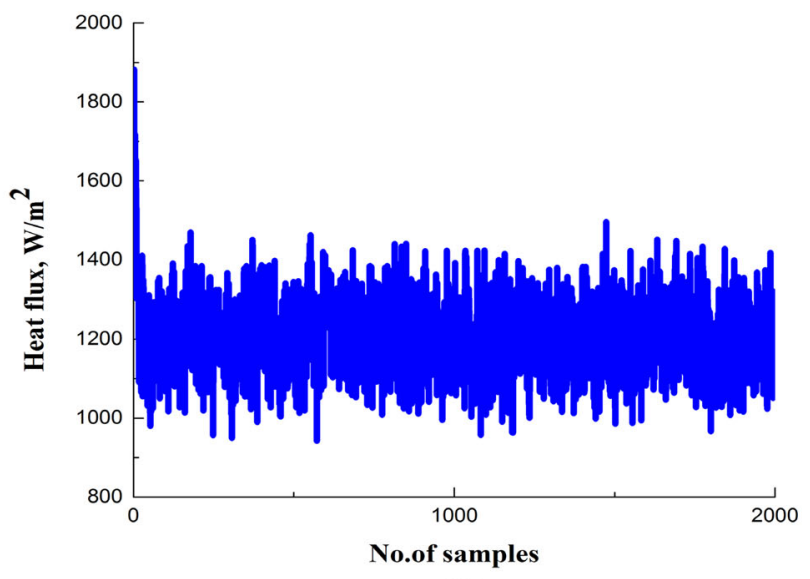

(b)

Figure 13. Sampling distribution for heat flux $1200 \mathrm{~W} / \mathrm{m}^{2}$ with initial guess $(\mathbf{a})=800 \mathrm{~W} / \mathrm{m}^{2}$ and $(\mathbf{b})=1800 \mathrm{~W} / \mathrm{m}^{2}$.

There is no remarkable difference in the values estimated by using 5000 samples and 10,000 samples. Hence, to reduce the computational cost and time, the number of samples used in this analysis is 5000 . The concept of burn in has been adopted to avoid the influence of initial guess, that is, first 1000 samples are not considered to calculate mean, MAP and SD. The entire estimation procedure is repeated for different heat flux and number sample considered is 5000. In this way, the robustness of the Bayesian framework along with MH-MCMC algorithm proves to be potent tool for the estimation of boundary heat flux for the conjugate heat transfer problem. Figure 12 shows the PPDF of the retrieved values of heat flux with uniform prior and normal prior, respectively.

The PPDF gives information about mean and MAP of the estimated parameter. Figure 13 shows the sampling distribution of heat flux $1200 \mathrm{~W} / \mathrm{m}^{2}$. An initial guess of $800 \mathrm{~W} /$ $\mathrm{m}^{2}$ and $1800 \mathrm{~W} / \mathrm{m}^{2}$ is assumed as different cases and the solution quickly converges to the actual heat flux of $1200 \mathrm{~W} / \mathrm{m}^{2}$ within 500 samples, which is evident from figure 13(a) and (b) respectively. Irrespective of the initial guess, the proposed algorithm reaches the solution at a faster rate.

\subsection{Heat flux retrieval for perturbed data}

In reality, the temperature distribution cannot be pure and the presence of random noise can be observed when experiments are performed. Nevertheless, the measurement data are contaminated with Gaussian errors that can cause large deviations of the final solution from the exact solution. To mimic experimental data, the surrogated data are now added with noise at various levels. The forward model is solved for the known heat flux and the temperature distribution obtained is perturbed by adding Gaussian noise such as $0.1 \mathrm{~K}, 0.5 \mathrm{~K}$. This represents noisy data similar to the experimental temperature. So, one is interested not only in estimation of unknown properties for surrogated data, but also in how the estimation can be applied for noisy data. The estimation results with $0.1 \mathrm{~K}$ and $0.5 \mathrm{~K}$ noise in the data are shown in table 9.

With the noise level of $(\sigma=0.5 \mathrm{~K})$, which in terms of measurement level will be $3 \sigma(1.5 \mathrm{~K})$, the error in the estimation is $1.2 \%$. When prior information about the parameter is incorporated, even with the noise addition the estimated values are much closer to the actual value than compared with the estimated values obtained from uniform prior. Hence, with the proper selection of the mean and the standard deviation of the prior, one can estimate the unknown parameters precisely. Once again, it has been proved that the potent Bayesian framework extended to estimation of the heat flux for the noise added surrogated data shows successful estimates and the retrieved heat flux deviates only marginally to the original value. The PPDF plot for the perturbed data for both uniform prior and normal prior at noise levels $0.1 \mathrm{~K}$ and $0.5 \mathrm{~K}$ is shown in figures 14 and 15 respectively. When normal prior is used for estimation the estimated values are closer to the actual value, whereas the value obtained from uniform prior is slightly deviated from the actual value, signifying that when non-informative prior is used then it is very much essential to reduce the measurement error. This helps improve the accuracy of the estimate. It has also been observed in table 9 that the standard deviation of the estimates using normal prior is lesser than the uniform prior for all heat fluxes. This is due to the a priori information of the unknown parameter, which simplifies the Markov chain process.

For the incorporation of Gaussian prior the standard deviation is considered as $\left\{\sigma_{\mathrm{p}}=0.05,0.06,0.09\right\}$ of the Gaussian mean. Different combinations of Gaussian mean 


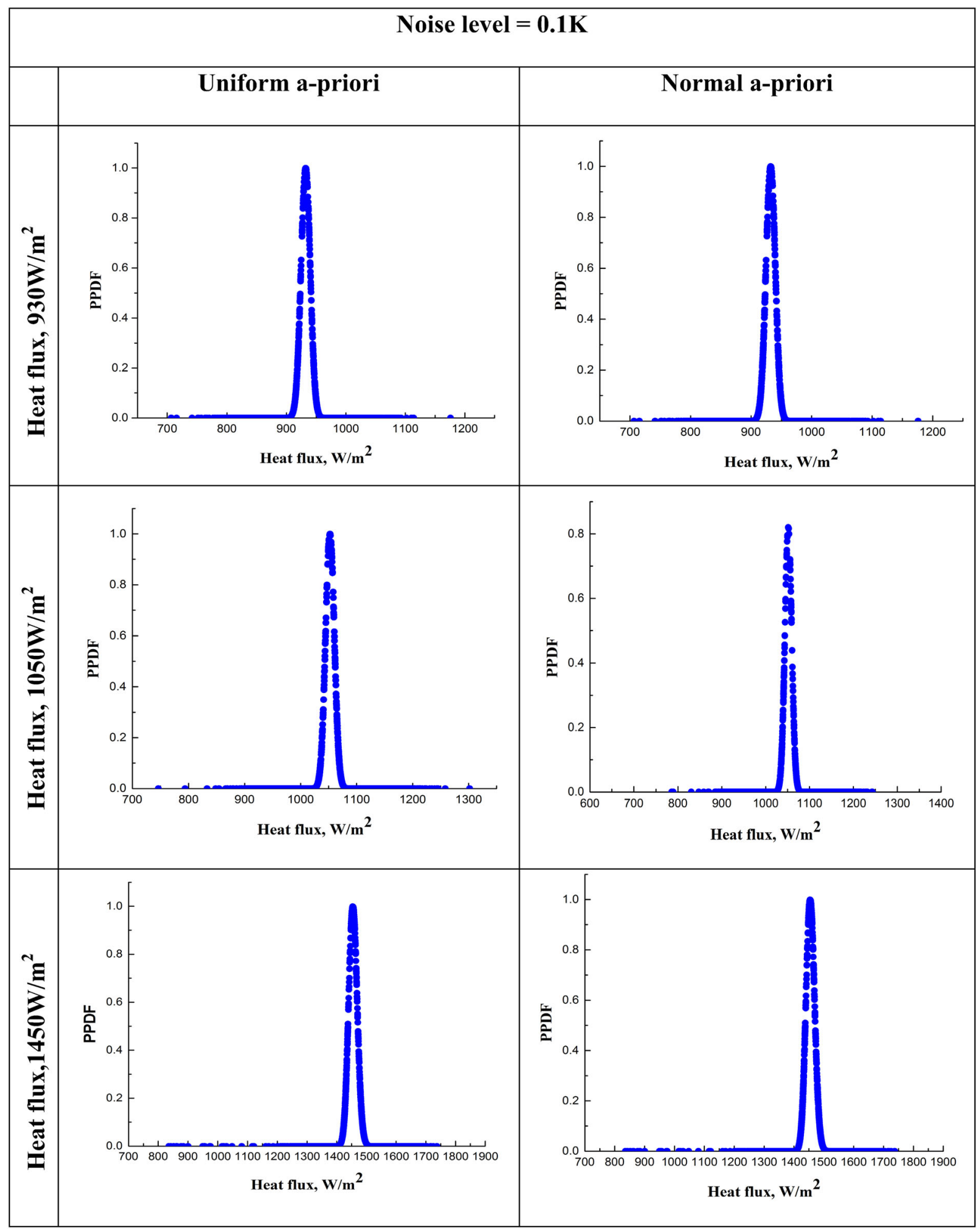

Figure 14. $\mathrm{PPDF}$ at noise level $=0.1 \mathrm{~K}$.

and standard deviation are considered. From table 10 it has been observed that proper selection of the mean and the standard deviation for the Gaussian distribution can estimate the values precisely. Table 10 shows that when $\sigma_{\mathrm{p}}$ $=0.09 \mathrm{x} \mu_{\mathrm{p}}$, the mean estimate is closer to the actual value of the heat flux. For the heat flux of $930 \mathrm{~W} / \mathrm{m}^{2}$, several 


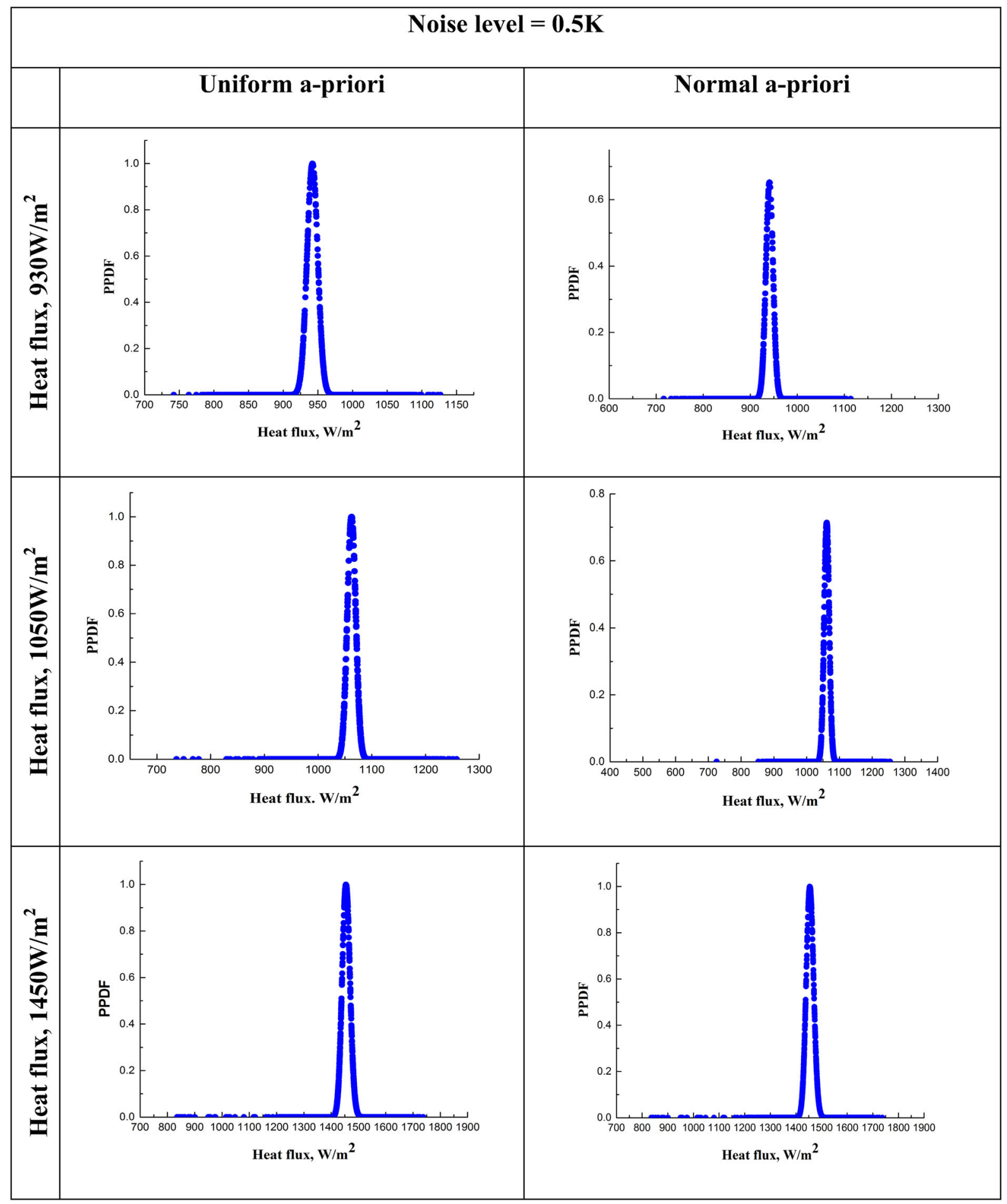

Figure 15. $\mathrm{PPDF}$ at noise level $=0.5 \mathrm{~K}$.

combinations of mean and standard deviation are used and it was observed that when the mean value was below $700 \mathrm{~W} / \mathrm{m}^{2}$ the estimated values are deviating from the actual values. Figure 16 shows the comparison of PPDF obtained for heat flux value of $1450 \mathrm{~W} / \mathrm{m}^{2}$ at $0.1 \mathrm{~K}$ noise levels, when uniform and normal priors are used during estimation. It is clearly observed that the standard deviation of the PPDF for normal prior is lesser than that of the standard deviation of the PPDF for uniform prior. This strongly proves the fact that incorporating prior information 
Table 10. Estimation of heat flux using the Gaussian prior.

\begin{tabular}{|c|c|c|c|c|c|}
\hline \multirow[b]{2}{*}{ Heat flux, W/m² } & \multicolumn{2}{|c|}{ Gaussian prior } & \multicolumn{3}{|c|}{$\mathrm{q}\left(\mathrm{W} / \mathrm{m}^{2}\right)$, retrieved } \\
\hline & $\mu_{p}(q)$ & $\sigma_{p}(q)$ & Mean & MAP & SD \\
\hline \multirow[t]{3}{*}{930} & 850 & 45.5 & 926.65 & 927.33 & 0.270 \\
\hline & 850 & 51 & 927.90 & 928.75 & 0.320 \\
\hline & 850 & 76.5 & 928.61 & 929.35 & 0.308 \\
\hline \multirow[t]{3}{*}{1050} & 950 & 47.5 & 1045.9 & 1047.6 & 0.321 \\
\hline & 950 & 57 & 1046.0 & 1048.0 & 0.313 \\
\hline & 950 & 85.5 & 1048.4 & 1048.7 & 0.326 \\
\hline \multirow[t]{3}{*}{1450} & 1350 & 65 & 1445.1 & 1445.6 & 0.443 \\
\hline & 1350 & 78 & 1446.3 & 1447.1 & 0.440 \\
\hline & 1350 & 117 & 1448.3 & 1448.6 & 0.472 \\
\hline
\end{tabular}

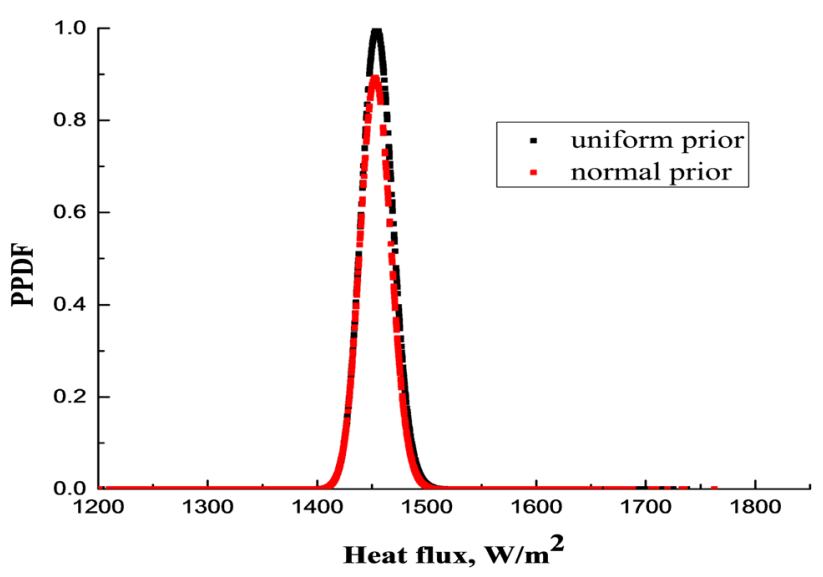

Figure 16. Comparison of PPDF for $1450 \mathrm{~W} / \mathrm{m}^{2}$ at $0.1 \mathrm{~K}$ noise level.

in the Bayesian framework will result in minimising the uncertainty associated with the estimated parameters.

\section{Conclusions}

A two-dimensional laminar conjugate natural convection from a fin with constant heat flux at the base has been considered for the present study. ANSYS14.5 fluent is used to model the computational geometry and simulations have been carried out successfully with the selected grid. Temperature distribution was obtained using CFD (forward model). A neural network is developed using ANN, and it acts as the fast forward model to obtain temperature distribution for various heat fluxes. A maximum error of $0.90 \%$ has been observed between the temperature obtained from CFD and ANN. Hence, CFD is replaced by ANN as the forward model. The Bayesian inference method was successfully implemented to estimate the unknown heat flux. The MCMC with MH algorithm has been explored to estimate the heat flux for the surrogated data. The unknown heat flux was also retrieved for the perturbed surrogated data with good accuracy. Gaussian prior was incorporated, and with the proper selection of the prior value, it was possible to obtain precise estimation of the parameters. The numerical results indicates that "Fast Forward ModelANN" approach can reduce the computational cost and the powerful Bayesian statistical inference results in better estimation process and quantifies the uncertainty accurately. Therefore, Bayesian inference can be successfully used for the estimation of parameters even for noisy data.

\section{Nomenclature}

ACFD asymptotic computational fluid dynamics

ANN artificial neural network

h heat transfer coefficient, $W / \mathrm{m}^{2} \mathrm{~K}$

$\mathrm{k}$ thermal conductivity of the fin material, $\mathrm{W} / \mathrm{m} \mathrm{K}$

L height of the fin, $m$

$\mathrm{p} \quad$ perimeter of the fin, $\mathrm{m}$

$\mathrm{q}_{\text {ref }} \quad$ reference heat flux, $\mathrm{W} / \mathrm{m}^{2}$

q flux input, $\mathrm{W} / \mathrm{m}^{2}$

$\mathrm{T}_{\text {ref }} \quad$ reference temperature, $\mathrm{K}$

$\mathrm{T}_{\text {new }}$ temperature for given value of heat flux, $\mathrm{K}$

$\mathrm{X}$ non-dimensional length

$\mathrm{x}$ distance from the base of fin, $\mathrm{m}$

\section{Greek symbols}

$\theta$ non-dimensional temperature

$\beta \quad 1 / T$, thermal expansion coefficient $\mathrm{K}^{-1}$

$\rho$ density, $\mathrm{kg} / \mathrm{m}^{3}$

$v$ kinematic viscosity, $\mathrm{m}^{2} / \mathrm{s}$

\section{References}

[1] Beck J V, Blackwell B and Clair C S 1985 Inverse heat conduction: ill-posed problems. New York: Wiley

[2] Ozisik M N and Orlande H R B 2000 Inverse heat transfer: fundamentals and applications. New York: Taylor and Francis

[3] Mota C A A, Orlande H R B, Carvalho M O M D, Kolehaminen V and J P Kaipio 2010 Bayesian estimation of 
temperature-dependent thermophysical properties and transient boundary heat flux. Heat Transfer Eng. 31:570-580

[4] Wang J and Zabaras N 2004 A Bayesian inference approach to the inverse heat conduction problem. Int. J. Heat Mass Transfer 47: 3927-3941

[5] Giralomi M 2008 Bayesian inference for differential equations. Theor. Comput. Sci. 408: 4-16

[6] Liang Yan, Fenglian Yang and Chuli Fu 2009 A Bayesian inference approach to identify a Robin coefficient in onedimensional parabolic problems. J. Comput. Appl. Math. 231:840-850

[7] Parthasarathy S and Balaji C 2008 Estimation of parameters in multi-mode heat transfer problems using Bayesian inference-effect of noise and a prior. Int. J. Heat Mass Transfer 51: 2313-2334

[8] Gnanasekaran N and Balaji C 2011 A Bayesian approach for the simultaneous estimation of surface heat transfer coefficient and thermal conductivity from steady state experiments on fins. Int. J. Heat Mass Transfer 54: 3060-3068

[9] Konda Reddy B, Gnanasekaran N and Balaji C 2012 Estimation of thermo-physical and transport properties with Bayesian inference using transient liquid crystal thermography experiments. J. Physics: Conference Series 395: 012082

[10] Cheung S H and Beck J L 2009 Bayesian model updating using hybrid Monte Carlo simulation with application to structural dynamic models with many uncertain parameters. J. Eng. Mech. 135: 243-255

[11] Gnanasekaran N and Balaji C 2013 Markov Chain Monte Carlo (MCMC) approach for the determination of thermal diffusivity using transient fin heat transfer experiments. Int. J. Therm. Sci. 63: 46-54

[12] Balaji C. and Tamanna Padhi 2010 A new ANN driven MCMC method for multi-parameter estimation in two-dimensional conduction with heat generation. Int. J. Heat Mass Transfer 53: 5440-5455

[13] Deng S and Hwang Y 2006 Applying neural networks to the solution of forward and inverse heat conduction problems. Int. J. Heat Mass Transfer 49: 4732-4750

[14] Ghadimi B, Kowsary F and Khorami M 2015 Heat flux online estimation in a locomotive brake disc using artificial neural networks. Int. J. Therm. Sci. 90: 203-213

[15] Somasundharam S and Reddy K S 2016 Inverse estimation of thermal properties using Bayesian inference and three different sampling techniques. Inverse Probl. Sci. Eng. https://doi.org/10.1080/17415977.2016.1138946

[16] Cole K D, Tarawneh C and Wilson B 2009 Analysis of fluxbase fins for estimation of heat transfer coefficient. Int. J. Heat Mass Transfer 52: 92-99

[17] Cole K D, Beck J V, Keith A Woodbury and Filippo de Monte 2014 Intrinsic verification and a heat conduction database. Int. J. Therm. Sci. 78: 36-47

[18] Prietob D, Asensioa M I, Ferraguta L and Cascón J M 2015 Sensitivity analysis and parameter adjustment in a simplified physical wildland firemodel. Adv. Eng. Softw. 90: 98-106

[19] Pereyra S, Lombera G, Frontini G and Urquiza S A 2014 Sensitivity analysis and parameter estimation of heat transfer and material flow models in friction stir welding. Mater. Res. 17(2): 397-404

[20] Cheng-Hung Huang, Li-Chun Jan, Rui Li and Albert J Shih 2007 A three-dimensional inverse problem in estimating the applied heat flux of a titanium drilling - theoretical and experimental studies. Int. J. Heat Mass Transfer 50: 3265-3277

[21] Huang C H and Chen W C 2000 A three-dimensional inverse forced convection problem in estimating surface heat flux by conjugate gradient method. Int. J. Heat Mass Transfer 43(17): 3171-3181

[22] Chen T C, Liu C C, Jang H Y and Tuan P C 2007 Inverse estimation of heat flux and temperature in multi-layer gun barrel. Int. J. Heat Mass Transfer 50(11-12): 2060-2068

[23] Wikstrom P, Blasiak W and Berntsson F 2007 Estimation of the transient surface temperature and heat flux of a steel slab using an inverse method. Appl. Therm. Eng. 27(14-15): 2463-2472

[24] Cui Miao, Yang Kai, Liu Yun-fei and Gao Xiao-wei 2012 Inverse estimation of transient heat flux to slab surface. $J$. Iron Steel Res. Int. 19(11): 13-18

[25] Man Young Kim 2007 A heat transfer model for the analysis of transient heating of the slab in a direct-fired walking beam type reheating furnace. Int. J. Heat Mass Transfer 50: 3740-3748

[26] Incropera F P and De Witt D P 2002 Fundamentals of heat and mass transfer. 5th edn. New York, NY: John Wiley \& Sons

[27] Wang $\mathbf{J}$ and Zabaras N 2005 Hierarchical Bayesian models for inverse problems in heat conduction. Inverse Probl. 21: 183-206 